\title{
Alarm-Related Workload in Default and Modified Alarm Settings and the Relationship Between Alarm Workload, Alarm Response Rate, and Care Provider Experience: Quantification and Comparison Study
}

Manikantan Shanmugham, MS; Lesley Strawderman, PhD; Kari Babski-Reeves, PhD; Linkan Bian, PhD

Department of Industrial and Systems Engineering, Mississippi State University, Mississippi State, MS, United States

Corresponding Author:

Manikantan Shanmugham, MS

Department of Industrial and Systems Engineering

Mississippi State University

260 McCain Hall

479-2 Hardy Road

Box 9542

Mississippi State, MS, 39762

United States

Phone: 18016739973

Email:

\section{Abstract}

Background: Delayed or no response to impending patient safety-related calls, poor care provider experience, low job satisfaction, and adverse events are all unwanted outcomes of alarm fatigue. Nurses often cite increases in alarm-related workload as a reason for alarm fatigue, which is a major contributor to the aforementioned unwanted outcomes. Increased workload affects both the care provider and the patient. No studies to date have evaluated the workload while caring for patients and managing alarms simultaneously and related it to the primary measures of alarm fatigue-alarm response rate and care provider experience. Many studies have assessed the effect of modifying the default alarm setting; however, studies on the perceived workload under different alarm settings are limited.

Objective: This study aimed to assess nurses' or assistants' perceived workload index of providing care under different clinical alarm settings and establish the relationship between perceived workload, alarm response rate, and care provider experience.

Methods: In a clinical simulator, 30 participants responded to alarms that occurred on a physiological monitor under 2 conditions (default and modified) for a given clinical condition. Participants completed a National Aeronautics and Space Administration-Task Load Index questionnaire and rated the demand experienced on a 20-point visual analog scale with low and high ratings. A correlational analysis was performed to assess the relationships between the perceived workload score, alarm response rate, and care provider experience.

Results: Participants experienced lower workloads when the clinical alarm threshold limits were modified according to patients' clinical conditions. The workload index was higher for the default alarm setting (57.60 [SD 2.59]) than for the modified alarm setting (52.39 [SD 2.29]), with a statistically significant difference of 5.21 (95\% CI 3.38-7.04), $t_{28}=5.838, P<.05$. Significant correlations were found between the workload index and alarm response rate. There was a strong negative correlation between alarm response rate and perceived workload, $\rho_{28}=-.54, P<.001$ with workload explaining $29 \%$ of the variation in alarm response rate. There was a moderate negative correlation between the experience reported during patient care and the perceived workload, $\rho_{28}=-.49, P<.05$.

Conclusions: The perceived workload index was comparatively lower with alarm settings modified for individual patient care than in an unmodified default clinical alarm setting. These findings demonstrate that the modification of clinical alarm limits positively affects the number of alarms accurately addressed, care providers' experience, and overall satisfaction. The findings support the removal of nonessential alarms based on patient conditions, which can help care providers address the remaining alarms accurately and provide better patient care. 
(JMIR Hum Factors 2018;5(4):e11704) doi: 10.2196/11704

\section{KEYWORDS}

clinical alarms; fatigue; physiologic monitoring; nursing; workload

\section{Introduction}

\section{Background}

Physiological monitor alarms and alerts specifically designed by medical device manufacturers are intended to alert clinicians to any deviation of physiological signals from the normal value. Although these devices ensure that doctors and nurses are always informed of physiological changes so as to respond to important deterioration events quickly, they generate very frequent alarms, of which a significant proportion are false [1-5]. Most of these alarms are not relevant to making clinical decisions, providing patient care, or ensuring patients' safety. About $70 \%$ of the alarms occurring in adult intensive care units do not add any value to the nurses' work process when monitoring patients [6].

Clinical alarms have received immense attention from clinicians, hospital administrators, and watchdog agencies, especially after the US Food and Drug Administration reported 566 alarm-related patient deaths $[7,8]$. The task of separating the true, actionable alarms from the false or nonactionable alarms lies with the clinicians responsible for responding to the alarms, who in most settings are nurses and their assistants. Alarm fatigue among health care workers, especially nurses, poses a risk to patient safety $[9,10]$. Upon deciding and initiating appropriate medical treatment, doctors hand off patients from their care to nurses and their assistants during recovery. Patients need to be continuously monitored during this recovery phase for any status changes [11]. When caring for multiple patients, nurses are exposed to numerous alarms per patient per shift and over time become fatigued due to an overwhelming number of alarms [12]. A frequently suggested solution to reduce fatigue is to adjust alarm parameters to suit patient conditions or a standard hospital protocol rather than using textbook normal values or default settings. However, the outcome of this suggestion was mixed $[13,14]$.

Several types of devices-infusion pumps, physiological monitors, and therapy delivery devices-are used in typical patient care settings, and multiple alarms from these devices can cause information overload, leading to clinical errors and poor overall patient outcomes. During clinical alarm management, nurses perform many activities that require excessive cognitive processing, which may contribute to sensory overload, and therefore, their alertness may decrease and errors may occur [15]. Particularly, mental overload may decrease the functioning of working memory. Therefore, assessing the mental workload of attending nurses while they operate these medical devices and monitor patients using physiological monitors is important. Although fatigue and workload are conceptually different, they are closely related. Some researchers have described alarm fatigue as a multicausal, multidimensional, nonspecific, and subjective phenomenon resulting from prolonged activity and psychological, socioeconomic, and environmental factors that affect both the mind and the body [16]. Therefore, assessing mental workload during alarm management will help understand alarm fatigue better. Nurses are an important resource who directly affect the health care system; therefore, ensuring optimal workload level is imperative [17].

\section{Objective}

Although several studies have reported that nurses' fatigue contributes to alarm mismanagement, no studies have quantified fatigue during alarm management and its effect on patient care quality and outcome. Little research has investigated workload and its correlation with alarm hazards and nurse response time. Given that clinical alarm management is a complex area in its infancy, cognitive workload cannot be described using 1 dimension or characteristic. A multidimensional scale is needed to quantify the mental workload. The National Aeronautics and Space Administration Task Load Index (NASA-TLX) provides a subjective measure of mental demand, physical demand, and temporal demand along with subjects' own performance, effort, and frustration [18]. Overall workload is measured by summing the scores on the 6 subscales. Although some studies have assessed mental workload in a clinical setting, the specific impact of increased workload on alarm management, response rate, and error rate has not been examined [19-21]. In subjective mental workload, the worker knows the amount of work needed to meet a particular demand. Subjective workload scales have been a familiar part of the human factors and ergonomics tool kit since the 1980s [22]. This study aimed to assess whether any changes in situational complexity, which is differentiated alarm settings, influence the subjective and physiological levels of mental workload and affect the care provider's experience while caring for patients.

\section{Methods}

\section{Design, Sample, and Setting}

The Mississippi State University's institutional review board approved this study, and participants' implied consent was obtained. This study was conducted in a clinical simulator. A total of 30 participants ( 23 females and 7 males) aged 24 to 60 years (mean 40.66 [SD 9.85]) were recruited. Participants were recruited from hospitals in the Pacific Northwest area of the western United States by word of mouth, phone calls, and flyer postings. Demographic data are presented in Table 1. Participants were randomly assigned to 1 of the 2 alarm threshold groups, default alarm setting and modified setting. Inclusion criteria for the study were medical alarm exposure and basic patient care experience. There were no exclusion criteria. The entire experiment was conducted in 2 waves over the course of 2 weeks. A week was allocated for each alarm setting-default alarm threshold and modified setting. The clinical simulator is equipped with modern physiological monitors and with intensive care equipment for life support, such as infusion and syringe pumps. The simulator setup for experiments was a progressive step-down care unit (patients in 
this unit are typically low-risk and in the recovery phase of their one-way mirror in the simulator, and data were recorded. clinical condition). The entire session was observed through a

Table 1. Demographic data.

\begin{tabular}{|c|c|}
\hline Variables & Statistics \\
\hline Age in years, mean $(\mathrm{SD})$ & $40.6(9.9)$ \\
\hline \multicolumn{2}{|l|}{ Gender, $\mathbf{n}(\%)$} \\
\hline Female & $23(77)$ \\
\hline Male & $7(23)$ \\
\hline \multicolumn{2}{|l|}{ Nursing background, n (\%) } \\
\hline Registered nurse & $10(33)$ \\
\hline Nurse assistants $\left(\mathrm{CNAs}^{\mathrm{a}}\right)$ & $20(67)$ \\
\hline \multicolumn{2}{|c|}{ Years of experience in managing device alarms, $n(\%)$} \\
\hline None & 0 \\
\hline Less than 1 year & $1(3)$ \\
\hline $1-3$ years & $3(10)$ \\
\hline $3-5$ years & $9(30)$ \\
\hline More than 5 years & $17(57)$ \\
\hline \multicolumn{2}{|c|}{ Trained on medical device alarms, $\mathbf{n}(\%)$} \\
\hline Yes & $10(33)$ \\
\hline No & $20(67)$ \\
\hline \multicolumn{2}{|c|}{ Training provided by your institution is adequate, $\mathbf{n}(\%)^{b}$} \\
\hline Yes & $5(17)$ \\
\hline No & $14(47)$ \\
\hline \multicolumn{2}{|c|}{ Did your assigned unit provide any training? $n(\%)^{b}$} \\
\hline Yes & $7(23)$ \\
\hline No & $8(27)$ \\
\hline \multicolumn{2}{|l|}{ Educational background, n (\%) } \\
\hline $\mathrm{CNAs}^{\mathrm{a}}$ or other & $20(67)$ \\
\hline Associates & $4(13)$ \\
\hline Bachelors & $4(13)$ \\
\hline Graduate and more & $2(7)$ \\
\hline \multicolumn{2}{|c|}{ Any other certifications? n $(\%)^{b}$} \\
\hline Yes & $5(17)$ \\
\hline No & $6(20)$ \\
\hline
\end{tabular}

${ }^{\mathrm{a}} \mathrm{CNA}$ : certified nursing assistant.

${ }^{\mathrm{b}}$ Percentage does not equal 100 due to missing responses.

\section{Procedure, Instrumentation, and Data Collection}

The patient condition to be monitored was kept constant to reduce variability. As previous studies have shown that a typical nurse in a progressive care unit does not spend their entire time solely on alarm management and performs other duties for up to 3 patients [23-25], a similar set up was reproduced in a clinical simulator for this experiment. In total, 3 male patient mannequins (SimMan), identified as M-1, M-2, and M-3, and considered low risk based on the Goldman risk chart, were placed in supine positions. M-1 was instrumented with a ProSim SpotLight pulse oximeter simulator (Fluke Bio, Bothell, WA). A physiological monitor (Nellcor with software algorithm Smart SatSec feature for customization) connected to the pulse oximeter simulator presented the alarms shown in Table 2 . The physiological monitor was set at default for the default setting portion of the experiment, and Smart SatSec was used for the modified setting. Alarms (shown in Table 2) were presented on 
the screen at a programmed time interval using auto sequence mode. For both settings, the software algorithm was programmed to keep the alarm available for 75 seconds and automatically stop when the time lapsed. M-2 and M-3 did not require monitoring; they were simply recovering from minor outpatient surgical procedures. These mannequins were included to emulate a progressive care unit as closely as possible. Participants performed other assigned dummy patient-care tasks on these mannequins as part of the experiment. The additional tasks are described in the following section. Participants were strongly encouraged to complete all dummy tasks. These tasks were also set at the same difficulty level between different alarm conditions (normal alarm threshold and modified setting) to minimize variability. No experimental data other than completion rates were recorded on these tasks. The independent variables were the 2 alarm settings, and the dependent variables were alarm response rate, care provider experience, and overall satisfaction. After providing their background and demographic information, participants rated their care provider experience and overall satisfaction on a 5-point Likert scale survey. Furthermore, the percentage of incorrectly addressed alarms out of the total number of addressed alarms, defined as the error rate, was computed and used as dependent variable.

\section{Various Alarms}

All types of alarms allowed by the physiologic monitor manufacturer were considered in this study. They are defined as follows. An actionable alarm is an alarm that requires a clinician's intervention or warrants a clinician's input or interaction with other clinicians or patients. This alarm should lead to immediate intervention, but due to alarm fatigue could go unwitnessed or misinterpreted by the attending clinician. Actionable alarms require timely intervention to prevent an adverse event. A nonactionable alarm correctly identifies the underlying patient's physiologic condition, but does not require intervention. Its validity is based on waveform quality and accuracy, strength of signals from leads and detectors, and artifact conditions. Transient low-oxygen saturation and heart rate alarms are a few examples of nonactionable alarms. System messages are notifications about medical devices or monitor condition and do not require clinical intervention. A notification about upcoming preventive maintenance of a device is an example for this category. Advisory alarms are status indicators about the parameters monitored and are nonactionable. Elapsed therapy time and amount of remaining fluids left to be delivered are examples for advisory alarms.

Table 2. Alarm sequence.

\begin{tabular}{|c|c|c|c|c|}
\hline \multirow[t]{2}{*}{ Serial no. } & \multicolumn{2}{|c|}{$\begin{array}{l}\text { Default setting of the alarm (as released to the hospital floor); } \\
\text { total number of alarms }=18\end{array}$} & \multicolumn{2}{|c|}{$\begin{array}{l}\text { Modified to patient condition using Smart SatSec; total number } \\
\text { of alarms }=11\end{array}$} \\
\hline & Alarm type & Intervention type & Alarm type & Intervention type \\
\hline 1 & Advisory & Nonactionable & Removed $^{\mathrm{a}}$ & Removed $^{\mathrm{a}}$ \\
\hline 2 & Warning & Actionable & Warning & Actionable \\
\hline 3 & System message & Nonactionable & Removed $^{\mathrm{a}}$ & Removed $^{\mathrm{a}}$ \\
\hline 4 & Actionable & Actionable & Actionable & Actionable \\
\hline 5 & Warning & Actionable & Warning & Actionable \\
\hline 6 & System message & Nonactionable & Removed $^{\mathrm{a}}$ & Removed $^{\mathrm{a}}$ \\
\hline 7 & Warning & Actionable & Warning & Actionable \\
\hline 8 & Actionable & Actionable & Actionable & Actionable \\
\hline 9 & Warning & Actionable & Warning & Actionable \\
\hline 10 & System message & Nonactionable & System message & Nonactionable \\
\hline 11 & System message & Nonactionable & Removed $^{\mathrm{a}}$ & Removed $^{\mathrm{a}}$ \\
\hline 12 & Advisory & Nonactionable & Removed $^{\mathrm{a}}$ & Removed $^{\mathrm{a}}$ \\
\hline 13 & Warning & Actionable & Warning & Actionable \\
\hline 14 & Advisory & Nonactionable & Advisory & Nonactionable \\
\hline 15 & Actionable & Actionable & Actionable & Actionable \\
\hline 16 & System message & Nonactionable & System message & Nonactionable \\
\hline 17 & Advisory & Nonactionable & Removed $^{\mathrm{a}}$ & Removed $^{\mathrm{a}}$ \\
\hline 18 & Advisory & Nonactionable & Removed $^{\mathrm{a}}$ & Removed $^{\mathrm{a}}$ \\
\hline
\end{tabular}

${ }^{\mathrm{a}}$ These alarms were not presented. Removed alarms: 5 premature ventricular contraction, 1 missed beat, and 1 noninvasive blood pressure. 


\section{Additional Task Details}

The calls were made through an intercom system from outside the simulator, and participants were prompted using the simulator voice communication system at the appropriate time to make calls. Completion rates of tasks in this session were recorded but were not analyzed. Participants were reminded through the microphone when the task was due for completion. To minimize order and interference effects, a 15-min warm-up period before starting the session and a 2-min cooling period between tasks were provided to participants. During the warm-up period, we discussed alarms and scenarios and asked them to respond verbally. As interference effects between tasks may impact participants' alarm management, tasks 1 to 4 were presented with a 2-min cooling period before and after:

1. Task 1: call Pharmacy and check the status of ordered medicine for patient mannequin \#2 (timing: 2 min into the experiment; call duration: 30 seconds)

2. Task 2: enter blood work result in Epic hospital system software for patient mannequin \#3 (timing: 10 min into the experiment; task duration: $2 \mathrm{~min}$ )

3. Task 3: administer a bolus dose of pain medicine for patient mannequin \#2 (timing: 14 min into the experiment; task duration: $1 \mathrm{~min}$ )

4. Task 4: take a call from another hospital unit to receive a patient into this unit (timing: 19 min into the experiment; task duration: $2 \mathrm{~min}$ ).

\section{Data Analysis}

Participant characteristics, number of alarms addressed, errors made during management, care provider experience, and overall satisfaction were described using descriptive statistics. To determine any significant differences between the mean alarm response and error rates, 2 one-way analysis of variances (ANOVAs) were performed. As the normality assumptions of the ANOVA were violated according to the Ryan-Joiner method, the Welch-ANOVA method was performed to test hypotheses. A Wilcoxon median rank within-subject test was used to identify any differences in care provider experience and participants' satisfaction levels when managing alarms in 2 different settings. Relationships between alarm workload and alarm response rate, error rate, care provider experience, and overall satisfaction were established using Spearman rank-order or Pearson product-correlation moment. $P<.05$ was considered statistically significant. IBM SPSS Version 25 for Windows was used for all statistical analyses.

\section{Results}

\section{Participant Characteristics}

Descriptive statistics for the dependent variables are shown in Table 3. A series of chi-square comparison tests were performed to examine whether the NASA-TLX subscale scores differed as a function of demographic characteristics (ie, age, gender, years of experience as a nurse, and alarm management experience). No differences were noted across all analyses $(P>.05)$.

\section{Workload Index}

An independent samples $t$ test was performed to determine any differences in participants' perceived workload between modified and default settings. An inspection of a boxplot indicated no outliers in the data. Workload index scores for each of the 6 subscales were normally distributed, as assessed by the Shapiro-Wilks test $(P>.05)$, and there was homogeneity of variances, as assessed by Levene test for equality of variances $(P=.18)$. The workload index was higher for the default alarm setting (57.60 [SD 2.59]) than for the modified alarm setting (52.39 [SD 2.29]), with a statistically significant difference of 5.21 (95\% CI 3.38-7.04), $t_{28}=5.838, P<.05$. Figure 1 shows participants' individual ratings on each subscale along with computed overall workload index.

Table 3. Descriptive statistics for dependent variables.

\begin{tabular}{|c|c|c|}
\hline Alarm setting and variable & Mean (SD) & Total \\
\hline \multicolumn{3}{|l|}{ Default } \\
\hline Percentage of alarms addressed & $68.9(10.5)$ & 30 \\
\hline Error rate & $9.5(6.0)$ & 30 \\
\hline Care provider experience ${ }^{\mathrm{a}}$ & $2.6(1.3)$ & 30 \\
\hline Overall satisfaction $^{\mathrm{a}}$ & $2.5(0.9)$ & 30 \\
\hline \multicolumn{3}{|l|}{ Modified } \\
\hline Percentage of alarms addressed & $86.7(7.6)$ & 30 \\
\hline Error rate & $2.6(4.5)$ & 30 \\
\hline Care provider experience ${ }^{\mathrm{a}}$ & $3.8(0.8)$ & 30 \\
\hline Overall satisfaction $^{\mathrm{a}}$ & $4.3(0.6)$ & 30 \\
\hline
\end{tabular}

${ }^{\mathrm{a}}$ Measured on 5-point Likert scale of 1-5 (1=very dissatisfied; 5=very satisfied). 
Figure 1. Subscale comparison chart for different alarm settings.

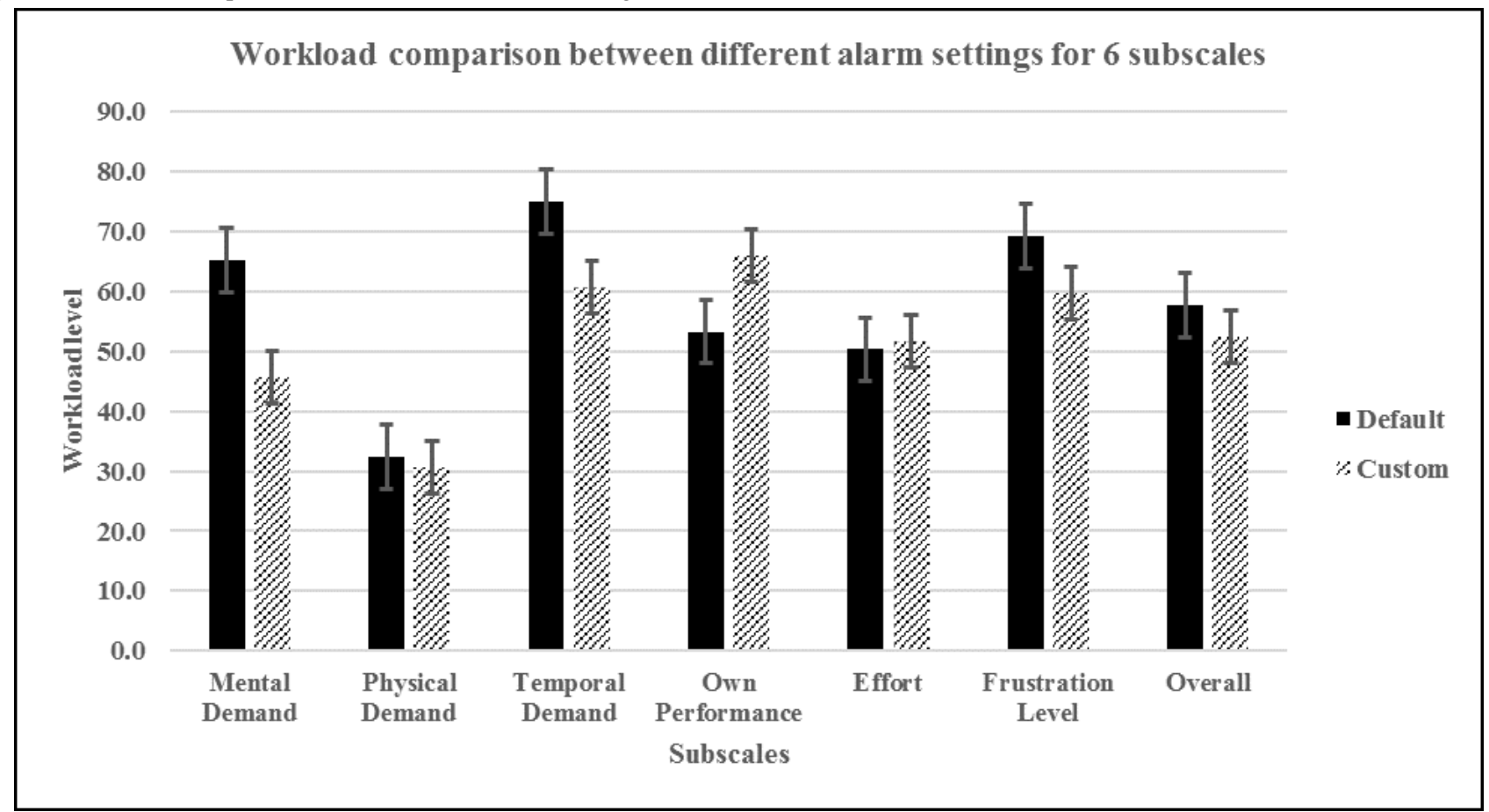

\section{Alarm Response Rate}

A one-way Welch ANOVA was performed to determine whether the alarm response rate was different for the 2 alarm threshold settings. Participants were classified into 2 groups: default $(n=15)$ setting and modified $(n=15)$ setting. Alarm response rate significantly differed between different alarm settings: Welch $F_{1,25.44}=29.05, P<.05$. Alarm response rate (ie, number of alarms addressed) increased from the default setting to the modified setting due to fewer alarms when physiological monitoring was modified to patient conditions.

\section{Relationship Between Alarm Workload and Alarm Response Rate}

Pearson product-moment correlation analysis was performed to assess the relationship between workload and the number of alarms addressed (alarm response rate) while providing patient care. The relationship was linear with both variables normally distributed, according to Shapiro-Wilks test $(P>.05)$, and there were no outliers. There was a strong negative correlation between alarm response rate and perceived workload, $\rho_{28}=-.54$, $P<.001$, with workload explaining $29 \%$ of the variation in alarm response rate. The negative correlation indicates that an increase in alarm workload is associated with a reduction in the number of addressed alarms; that is, modification of alarms according to patient conditions in patient-supporting medical devices help reduce care providers' workload and improve the alarm response rate.

\section{Error Rate}

A one-way Welch ANOVA was performed to determine whether the error rate was different for the default and modified settings. The error rate was significantly different between different alarm settings: Welch $F_{1,25.93}=12.46, P<.05$. The error rate significantly decreased from the default setting to the modified setting, primarily due to fewer alarms when physiological monitoring was modified to patient conditions.

\section{Relationship Between Alarm Workload and Error Rate}

A Spearman rank-order correlation analysis was performed to assess the relationship between alarm error rate and perceived workload while providing patient care. A visual inspection of a scatterplot showed a monotonic relationship. There was a strong positive correlation between the number of errors committed (alarm error rate) and the perceived workload, $\rho_{28}=.60, P<.05$. The number of errors committed by nurses or assistants dropped simultaneously with the corresponding workload, which shows that they are associated with each other in a health care environment.

\section{Care Provider Experience}

A Mann-Whitney U test was performed to determine whether there were differences in care provider experience between default and modified alarm settings. Distributions of care provider ratings for default and modified settings were similar, as assessed by visual inspection. Care provider experience ratings (on a 5-point Likert scale) for the modified setting (mean rank=20.83) were significantly higher than those for the default setting (mean rank=10.17), $U=32.5, z=-3.422, P=.001$, using an exact sampling distribution for $U$.

\section{Relationship Between Alarm Workload and Care Provider Experience}

A Spearman rank-order correlation analysis was performed to assess the relationship between perceived workload and care provider experience while providing patient care in a progressive care setting. A visual inspection of a scatterplot showed a monotonic relationship. There was a moderate negative correlation between the experience reported during patient care and the perceived workload, $\rho_{28}=-.49, P<.05$. The care provider 
experience, during or after caring for patients, was inversely proportional to the alarm-related workload. It is important to note that the participants were managing alarms along with several patient care tasks to mimic real-world situations. Therefore, any reduction in workload positively impacted care provider experience and well-being at the job.

\section{Overall Satisfaction}

To determine any differences in overall satisfaction between default and modified alarm settings, a Mann-Whitney $U$ test was performed. Distributions of overall satisfaction ratings for default and modified settings were similar, as assessed by visual inspection. Overall satisfaction ratings (on a 5-point Likert scale) for the modified setting (mean rank=21.90) were significantly higher than those for the default setting (mean rank=9.10), $U=16.5, z=-4.146, P=.001$, using an exact sampling distribution for $U$.

\section{Relationship Between Alarm Workload and Overall Satisfaction}

A Spearman rank-order correlation analysis was performed to assess the relationship between perceived workload and overall satisfaction while providing patient care in a progressive care setting. A visual inspection of a scatterplot showed a monotonic relationship. There was a strong negative correlation between the overall reported satisfaction and perceived workload, $\rho_{28}=-.69, P<.05$. The negative correlation indicates that the workload increase is associated with overall satisfaction, which decreased significantly. Therefore, hospital administrators and risk managers should consider customizing alarms in patient-supporting medical products, as it is a key factor of care providers' satisfaction.

\section{Discussion}

\section{Principal Findings}

Delayed or no response to impending patient safety-related calls, poor care provider experience, low job satisfaction, and adverse events are all unwanted outcomes of alarm fatigue. In this study, alteration of alarm limits by customizing the experimental settings based on patients' conditions resulted in lower NASA-TLX scores than those obtained using the default manufacturer settings. That is, allowing the physiological monitoring device to operate under a default setting based on normal textbook values resulted in more alarms, thereby leading to a higher mental workload while managing these alarms. Higher NASA-TLX scores indicate that alarm management is a complex task and has the potential to induce fatigue. Higher mental workload impacts nurses' attentiveness, increases the risk of slow responses, and can result in poor task accuracy. The number of alarm signals has been reported to reach several hundred per day for some patients in 1 study, thus creating a high alarm burden for nurses [26]. Nurses will be desensitized by such a high alarm burden and may miss, ignore, or disable alarm signals, which might result in adverse events [27].

The scores on NASA-TLX show that temporal demand, mental demand (MD), and frustration level are the major contributors to alarm workload. This is not surprising, as responding to alarms is secondary to primary care provider tasks such as medication administration, patient assessments, and note updates. In such dual-task systems, time spent on responding to alarms distracts from the primary tasks, and nurses feel pressed for time and frustrated. The higher MD score is attributable to the process involved in analyzing and isolating the source of the alarm, which often requires higher cognitive amplitude.

Participants' self-reported performance was higher in the modified setting than in the default setting. The higher alarm response rate in the modified setting supports this score. Better alarm response rate is also manifested across 2 other subscales, lower frustration and overall workload index, as shown in Figure 1. Not surprisingly, the subscale scores for physical demand and effort in the modified and default settings were statistically similar and lower compared with other subscales in their respective groups. Although only 4 of the $30(13 \%, 4 / 30)$ participants provided narrative data, making it difficult to generalize for the entire group, the common theme for the default setting was the excessive number of alarms and tasks. The most important finding is that the number of alarms addressed was inversely proportional to the workload encountered during patient care. Participants were able to address almost all presented alarms when the alarm settings were modified according to patient conditions. This finding is consistent with those of similar alarm setting modification studies, which showed that a $43 \%$ reduction in alarms is possible through alarm setting customization [26,27]. Participants also expressed positive views of alarm customization. Some researchers have reported reducing the total number of alarms from 180 per patient per day to 40 through a unit-level standardization project, which included a daily individualization of alarm parameters [28]. More than a 50\% reduction in the total rate of alarms per bed per day and a significant decrease in noise are possible by eliminating 3 types of ventricular contraction alarms [29].

Another unique finding of this study is that the alarm workload was directly proportional to the number of errors committed. The decrease in the number of errors is associated with the number of alarms that needed to be addressed during patient care. This suggests that the removal of certain nonessential alarms enabled the nurses to address the remaining important alarms accurately without any or with only minimal errors. The overwhelming number of alarms in the default setting put time pressure on nurses, and thus, they attempted to address more alarms within the limited time and made errors along the way. This can also be seen in a different way-if the number of opportunities (alarms) to make an error is limited, the number of errors committed will likely reduce.

Care provider experience and overall satisfaction were inversely correlated to alarm-related workload. As the alarm-related workload increases-which is typical when the alarms are set at the manufacturer's default setting - the quality of the experience of care providers caring for patients decreases. When the number of alarms to be assessed and addressed is low or lower they have more time to focus on patient care tasks and carry out other critical administrative tasks. The lesser the job stress and feeling of burn out, the higher the job satisfaction 
and general well-being in a typical health care setting [30]. It is likely that the lesser number of alarms in the modified setting allowed participants to complete all tasks with less time pressure and to be engaged with the system, which was reflected in higher satisfaction scores. The only difference between the default and modified experimental set-up(s) was the total number of alarms. Therefore, changes observed in care provider experience and overall satisfaction were most likely associated with modifications in alarm-related workload. A larger sample population and other types of monitoring devices are needed to determine whether alarm workload is the causal factor.

\section{Limitations}

The entire experiment was executed in a simulator lab setting, which is controlled and supported; therefore, the applicability of the findings should be examined further and may need to be repeated before being implemented into policies and procedures. Future studies should also include additional populations such as physicians, medical assistants, and other therapists who are also part of the patient care team. The sample population was entirely based out of 3 local hospitals in the Pacific Northwest region of the United States. It is well known that the health care field has regional cultures. Future studies should recruit participants across the country and investigate whether the effect of alarm modifications will bring similar benefits under other patient care settings such as intensive care, coronary care, emergency wards, and medical-surgical units.

\section{Conclusions}

The findings of this study show that removal of certain nonessential alarms based on patient condition can result in better care provider experience, reduced mental workload, and higher overall satisfaction. The number of managed alarms is directly proportional to workload and the number of errors (error rate) committed and inversely proportional to alarm response rate and care provider experience. Evidence for optimal alarm settings for physiological monitors and cardiac devices is abundant. Hospital administrators should make efforts to develop appropriate threshold levels for various physiological measures that clinicians monitor for typical patient conditions. This will help reduce the alarm burden for nurses and their aides significantly.

\section{Conflicts of Interest}

None declared.

\section{References}

1. Liu Y, Pecht MG. Reduction of motion artifacts in electrocardiogram monitoring using an optical sensor. Biomed Instrum Technol 2011 Mar;45(2):155-163. [doi: 10.2345/0899-8205-45.2.155] [Medline: 21466338]

2. Funk M, Stephens K, May J, Fennie K, Feder S, Drew B. An alarming rate of unnecessary monitoring in the Practical Use of the Latest Standards of Electrocardiography (PULSE) trial. J Am Coll Cardiol 2013 Mar 12;61(10):E1496 [FREE Full text] [doi: 10.1016/S0735-1097(13)61496-5]

3. Imhoff M, Kuhls S. Alarm algorithms in critical care monitoring. Anesth Analg 2006 May;102(5):1525-1537. [doi: 10.1213/01.ane.0000204385.01983.61] [Medline: 16632837]

4. Phillips J, Barnsteiner JH. Clinical alarms: improving efficiency and effectiveness. Crit Care Nurs Q 2005;28(4):317-323. [Medline: $\underline{16239820]}$

5. Atzema C, Schull MJ, Borgundvaag B, Slaughter GR, Lee CK. ALARMED: adverse events in low-risk patients with chest pain receiving continuous electrocardiographic monitoring in the emergency department. A pilot study. Am J Emerg Med 2006 Jan;24(1):62-67. [doi: 10.1016/j.ajem.2005.05.015] [Medline: 16338512]

6. Pergher AK, da Silva RC. [Stimulus-response time to invasive blood pressure alarms: implications for the safety of critical-care patients]. Rev Gaucha Enferm 2014 Jun;35(2):135-141 [FREE Full text] [Medline: 25158473]

7. Ruskin KJ, Hueske-Kraus D. Alarm fatigue: impacts on patient safety. Curr Opin Anaesthesiol 2015 Dec;28(6):685-690. [doi: 10.1097/ACO.0000000000000260] [Medline: 26539788]

8. The Joint Commission: Sentinel Event Alert. Jointcommission. 2013. Medical device alarm safety in hospitals URL: https:/ /www.jointcommission.org/assets/1/18/SEA 50 alarms 4513 FINAL1.PDF [accessed 2018-08-17] [WebCite Cache ID 71kQTUQeF]

9. Buist M, Bernard S, Nguyen TV, Moore G, Anderson J. Association between clinically abnormal observations and subsequent in-hospital mortality: a prospective study. Resuscitation 2004 Aug;62(2):137-141. [doi: 10.1016/j.resuscitation.2004.03.005] [Medline: 15294398]

10. Gazarian PK, Carrier N, Cohen R, Schram H, Shiromani S. A description of nurses' decision-making in managing electrocardiographic monitor alarms. J Clin Nurs 2015 Jan;24(1-2):151-159. [doi: 10.1111/jocn.12625] [Medline: 24813940]

11. Burgess LP, Herdman TH, Berg BW, Feaster WW, Hebsur S. Alarm limit settings for early warning systems to identify at-risk patients. J Adv Nurs 2009 Sep;65(9):1844-1852. [doi: 10.1111/j.1365-2648.2009.05048.x] [Medline: 19694847]

12. Gross B, Dahl D, Nielsen L. Physiologic monitoring alarm load on medical/surgical floors of a community hospital. Biomed Instrum Technol 2011;Suppl:29-36. [doi: 10.2345/0899-8205-45.s1.29] [Medline: 21599479]

13. Sowan AK, Gomez TM, Tarriela AF, Reed CC, Paper BM. Changes in default alarm settings and standard in-service are insufficient to improve alarm fatigue in an intensive care unit: a pilot project. JMIR Hum Factors 2016 Jan;3(1):e1 [FREE Full text] [doi: 10.2196/humanfactors.5098] [Medline: 27036170] 
14. Graham KC, Cvach M. Monitor alarm fatigue: standardizing use of physiological monitors and decreasing nuisance alarms. Am J Crit Care 2010 Jan;19(1):28-34 [FREE Full text] [doi: 10.4037/ajcc2010651] [Medline: 20045845]

15. Taenzer AH, Pyke JB, McGrath SP, Blike GT. Impact of pulse oximetry surveillance on rescue events and intensive care unit transfers: a before-and-after concurrence study. Anesthesiology 2010 Feb;112(2):282-287. [doi: 10.1097/ALN.0b013e3181ca7a9b] [Medline: 20098128]

16. Soh T, Crumpton LL. An evaluation of fatigue indicators while performing nursing tasks. 1996 May 15 Presented at: Industrial Engineering Research; May 15; Norcross, GA.

17. Zboril-Benson LR. Why nurses are calling in sick: the impact of health-care restructuring. Can J Nurs Res 2002 Mar;33(4):89-107. [Medline: 11998199]

18. Hart SG, Staveland LE. Development of NASA-TLX (Task Load Index): results of empirical and theoretical research. Adv Psychol 1988;52:139-183. [doi: 10.1016/S0166-4115(08)62386-9]

19. Hoonakker P, Carayon P, Gurses A, Brown R, McGuire K, Khunlertkit A, et al. Measuring workload of ICU nurses with a questionnaire survey: the NASA Task Load Index (TLX). IIE Trans Healthc Syst Eng 2011;1(2):131-143 [FREE Full text] [doi: 10.1080/19488300.2011.609524] [Medline: 22773941]

20. Yamase H. Development of a comprehensive scoring system to measure multifaceted nursing workloads in ICU. Nurs Health Sci 2003 Dec;5(4):299-308. [Medline: 14622382]

21. Carayon P, Gürses AP. A human factors engineering conceptual framework of nursing workload and patient safety in intensive care units. Intensive Crit Care Nurs 2005 Oct;21(5):284-301. [doi: 10.1016/j.iccn.2004.12.003] [Medline: 16182125]

22. Tsang PS, Vidulich MA. Mental workload and situation awareness. In: Salvendy G, editor. Handbook of Human Factors and Ergonomics. 3rd edition. Hoboken, NJ: John Wiley and Sons; 2011:243-268.

23. Spence Laschinger HK, Leiter MP. The impact of nursing work environments on patient safety outcomes: the mediating role of burnout/engagement. J Nurs Adm 2006 May;36(5):259-267. [Medline: 16705307]

24. Clark TJ, Yoder-Wise PS. Enhancing trifocal leadership practices using simulation in a pediatric charge nurse orientation program. J Contin Educ Nurs 2015 Jul 01;46(7):311-317. [doi: 10.3928/00220124-20150619-02] [Medline: 26154673]

25. Falk AC, Wallin EM. Quality of patient care in the critical care unit in relation to nurse patient ratio: a descriptive study. Intensive Crit Care Nurs 2016 Aug;35:74-79. [doi: 10.1016/j.iccn.2016.01.002] [Medline: 27117560]

26. Cvach MM, Biggs M, Rothwell KJ, Charles-Hudson C. Daily electrode change and effect on cardiac monitor alarms: an evidence-based practice approach. J Nurs Care Qual 2013;28(3):265-271. [doi: 10.1097/NCQ.0b013e31827993bc] [Medline: 23187092]

27. Cvach M. Monitor alarm fatigue: an integrative review. Biomed Instrum Technol 2012;46(4):268-277. [doi: 10.2345/0899-8205-46.4.268] [Medline: 22839984]

28. Dandoy CE, Davies SM, Flesch L, Hayward M, Koons C, Coleman K, et al. A team-based approach to reducing cardiac monitor alarms. Pediatrics 2014 Dec;134(6):e1686-e1694 [FREE Full text] [doi: 10.1542/peds.2014-1162] [Medline: 25384493]

29. Srinivasa E, Mankoo J, Kerr C. An evidence-based approach to reducing cardiac telemetry alarm fatigue. Worldviews Evid Based Nurs 2017 Aug;14(4):265-273. [doi: 10.1111/wvn.12200] [Medline: 28432853]

30. Young G, Zavelina L, Hooper V. Assessment of workload using NASA Task Load Index in perianesthesia nursing. J Perianesth Nurs 2008 Apr;23(2):102-110. [doi: 10.1016/j.jopan.2008.01.008] [Medline: 18362006]

\author{
Abbreviations \\ ANOVA: analysis of variance \\ MD: mental demand \\ NASA-TLX: National Aeronautics and Space Administration-Task Load Index
}

Edited by $G$ Eysenbach; submitted 26.07.18; peer-reviewed by M Cvach, SW Bae; comments to author 16.08.18; revised version
received 10.09.18; accepted 23.09.18; published 23.10.18
Please cite as:
Shanmugham M, Strawderman L, Babski-Reeves K, Bian L
Alarm-Related Workload in Default and Modified Alarm Settings and the Relationship Between Alarm Workload, Alarm Response
Rate, and Care Provider Experience: Quantification and Comparison Study
JMIR Hum Factors $2018 ; 5(4):$ e11704
URL: $\underline{\text { http://humanfactors.jmir.org/2018/4/e11704/ }}$
doi: $\underline{10.2196 / 11704}$
PMID: $\underline{30355550}$


(CManikantan Shanmugham, Lesley Strawderman, Kari Babski-Reeves, Linkan Bian. Originally published in JMIR Human Factors (http://humanfactors.jmir.org), 23.10.2018. This is an open-access article distributed under the terms of the Creative Commons Attribution License (https://creativecommons.org/licenses/by/4.0/), which permits unrestricted use, distribution, and reproduction in any medium, provided the original work, first published in JMIR Human Factors, is properly cited. The complete bibliographic information, a link to the original publication on http://humanfactors.jmir.org, as well as this copyright and license information must be included. 